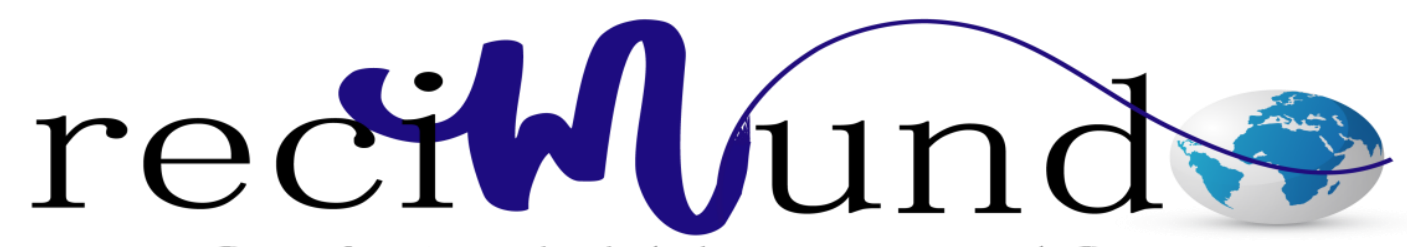

Revista Científica Mundo de la Investigación y el Conocimiento

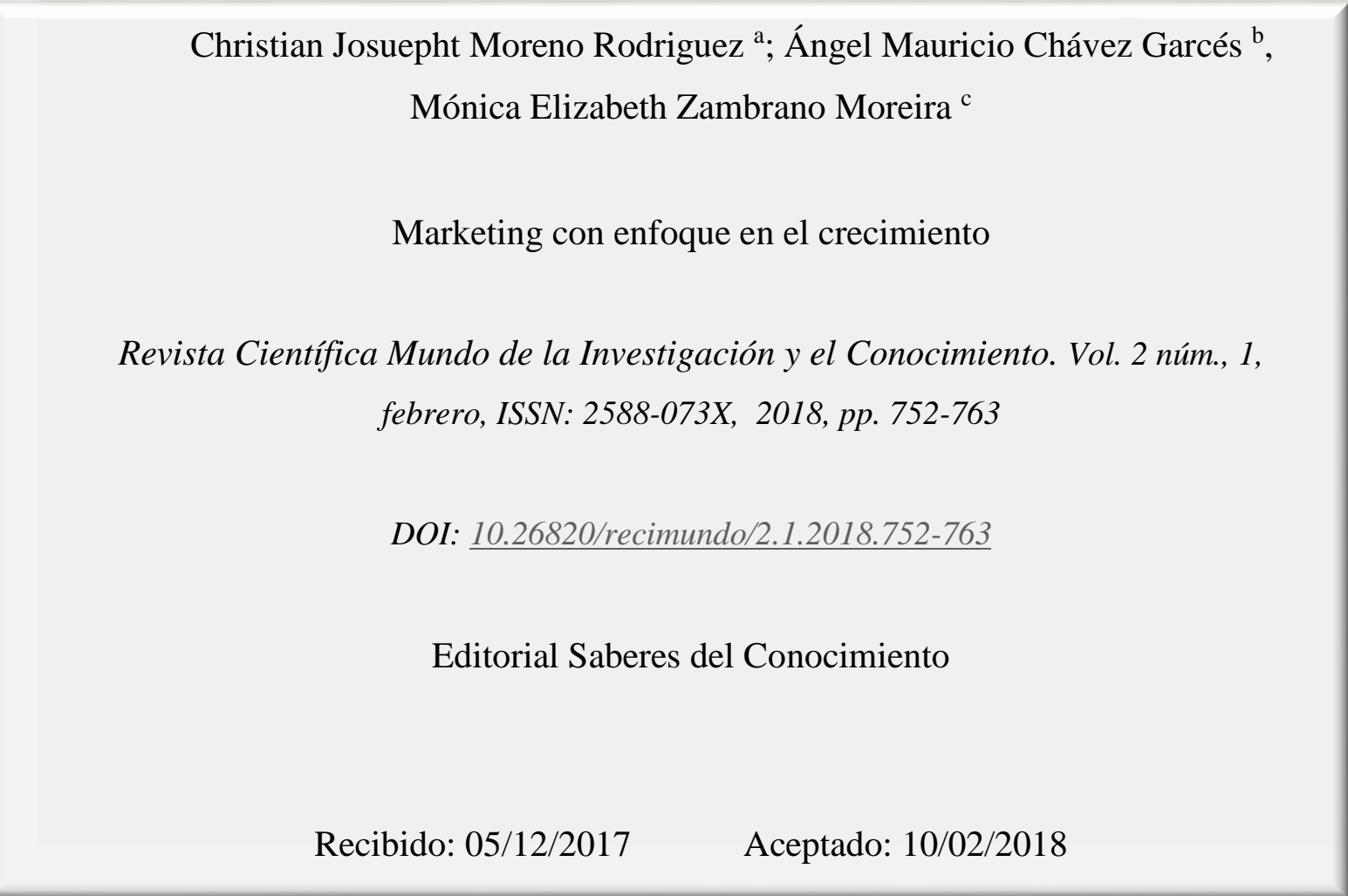

I. Universidad de Guayaquil; christian.morenorod@ug.edu.ec

II. Universidad de Guayaquil; angel.chavezga@ug.edu.ec

III. Universidad de Guayaquil; monica.zambranomo@ug.edu.ec 


\section{Marketing con enfoque en el crecimiento}

Vol. 2, núm. 1., (2018)

Christian Josuepht Moreno Rodriguez; Ángel Mauricio Chávez Garcés, Mónica Elizabeth Zambrano Moreira

\section{Resumen}

En los últimos tiempos en que se han activado el otorgamiento de créditos por parte de las entidades, el comercio necesita del crédito como instrumento generador del crecimiento. El conocimiento del cliente y la fidelización se muestran como una apuesta segura de crecimiento actual y futuro. Para lograrlo nos apoyamos en la tecnología ya que nos permite transformar la información en conocimiento abriendo las puertas de las posibilidades de negocios, pues sin conocimiento no pueden existir estrategias. $\mathrm{Y}$ es precisamente de aquí de donde surge el marketing dinámico, que nos permite explorar a través de la tecnología dichos conocimientos convirtiéndolo en oportunidades de venta.

En los últimos años el termino MARKETING ha logrado proyectar un gran impacto en el crecimiento económico logrando analizar la gestión comercial de las empresas con el fin de captar retener y fidelizar a los clientes y es gracias a este poderoso instrumento que las empresas obtienen grandes dividendos anuales superando hasta sus propias expectativas de venta

Palabras Claves: Marketing, crecimiento, impacto, global, enfoque. 


\section{Marketing con enfoque en el crecimiento}

Vol. 2, núm. 1., (2018)

Christian Josuepht Moreno Rodriguez; Ángel Mauricio Chávez Garcés, Mónica Elizabeth Zambrano Moreira

\section{Summary}

In recent times, when the granting of credits by banks has been activated, trade needs credit as a growth-generating instrument. Customer knowledge and loyalty are shown as a safe bet for real and future growth. To achieve this, we rely on technology that allows us to transform information into the knowledge of the doors of business possibilities, because without knowledge there can be no strategies. And this is precisely where dynamic marketing comes from, which allows us to explore through technology the knowledge that becomes sales opportunities.

In recent years the term MARKETING has managed to project a great impact on economic growth by analyzing the commercial management of companies in order to attract and retain customers and thanks to this powerful tool that companies obtain large annual dividends exceeding your own sales expectations

Keywords: Marketing, growth, global, impact, approach. 


\section{Marketing con enfoque en el crecimiento}

Vol. 2, núm. 1., (2018)

Christian Josuepht Moreno Rodriguez; Ángel Mauricio Chávez Garcés, Mónica Elizabeth Zambrano Moreira

\section{Introducción.}

El marketing no es más que la disciplina dedicada al análisis del comportamiento de los mercados y sus consumidores mientras que el crecimiento económico se refiere al incremento de ciertos indicadores, como la producción de bienes y servicios, el mayor consumo de energía, el ahorro, la inversión, una Balanza comercial favorable, el aumento de consumo de calorías per cápita, etc. El mejoramiento de éstos indicadores debería llevar teóricamente a un alza en los estándares de vida de la población.

Si fusionamos el marketing con el crecimiento global de una nación nos encontramos con que éste cumple un papel sumamente importante en la vida tanto en el consumo de productos como en el enfoque o venta de una idea que nos ayude a entender que una sociedad tiene un gran crecimiento a nivel económico y social.

Es por ello que en los últimos años la palabra marketing se ha convertido en una de los conceptos más utilizados en el ámbito empresarial abarcando también un sinfín de áreas en las que se relaciona, a diario escuchamos alguna noticia o acontecimiento en el que se utiliza el concepto de marketing. (Díaz \& Rubio, 2010)

Cuando nos referimos a marketing estamos hablando básicamente de una relación de intercambio donde su alcance no es exclusivamente empresarial este concepto abarca toda acción en donde se deba concebir una relación de intercambio que se centra en el reconocimiento y satisfacción de las exigencias y anhelos de los consumidores colocando como pieza más importante al cliente.

Un ejemplo son los partidos políticos quienes desarrollan una relación de intercambio con sus votantes. En dichos partidos los políticos ofrecen a su electorado una ideología y unas promesas a cambio de votos. Esta relación de intercambio también es objeto del estudio del marketing (marketing político y electoral) (Díaz \& Rubio, 2010) 


\section{Marketing con enfoque en el crecimiento}

Vol. 2, núm. 1., (2018)

Christian Josuepht Moreno Rodriguez; Ángel Mauricio Chávez Garcés, Mónica Elizabeth Zambrano Moreira

El marketing por parte de las empresas ha tenido un gran efecto en la economía global en las últimas décadas. El auge de la era de la computación ha logrado la aplicación de sistemas, funciones y actividades que permiten centrar la atención en el crecimiento de la economía mundial.

En los años 1980 las computadoras comenzaron a ser indispensables para cada individuo y su acceso a comprarlas se hizo más factibles lo que permitió abrir las puertas a la tecnología y al internet logrando mejoras en la comercialización de cualquier producto e impulsando el crecimiento económico.

Las redes sociales también representan un punto importante en el crecimiento con ellas puede manejarse el estudio de los mercados y la publicidad directamente al consumidor o la venta de alguna idea o producto de una manera casi inmediata, con tan solo utilizar herramientas tecnológicas tenemos acceso a toda clase de información por lo que el internet es pieza clave en cuanto al crecimiento.

Dentro del campo empresarial el marketing representa un papel indispensable. Desde la óptica fisiológica del negocio se entiende como una técnica de gestión para la captación, anticipación y satisfacción de las necesidades del cliente de forma rentable para la organización o empresa. (Cerveró, Iglesias, \& Villacampa, 2002). En este orden, Colbert y Cuadrado (2003:20) señalan que, el objetivo esencial del marketing no es otro que la optimización de la relación entre empresas y clientes y la maximización de su satisfacción mutua. (Anato, 2006)

Las funciones principales de la actividad de marketing (Cerveró et al(2002), son: Investigación; Innovación y creación de nuevos productos y servicios; Determinación de los precios; Intermediación o distribución de los servicios; Comunicación; Marketing interno, y Planificación.

Cada de una de estas etapas se integran en un Plan de Marketing que sirve de orientación a las organizaciones para gestionar las actividades de marketing de manera estructurada durante un período de tiempo considerando el análisis, el diagnóstico, los objetivos, las estrategias, las 


\section{Marketing con enfoque en el crecimiento}

Vol. 2, núm. 1., (2018)

Christian Josuepht Moreno Rodriguez; Ángel Mauricio Chávez Garcés,

Mónica Elizabeth Zambrano Moreira

actuaciones, el seguimiento y el control (Cerveró 2002: 27-28). Una vez establecidas las estrategias de actuación el siguiente paso consiste en la determinación de las actuaciones que por lo general se estructuran en cuatro líneas globales o en los denominados factores clásicos del marketing-mix o las cuatro $P$, como las definió McCarthy (1960 citado por Chaffey et al. 2000: 40; Colber y Cuadrado 2003:23): producto, precio, distribución y comunicación.

\section{Materiales y Métodos}

Esta investigación está enfocada en el estudio del impacto que ha tenido el marketing en el crecimiento económico a través de la recopilación de información importante que direccionan al conocimiento del enfoque del mercado a nivel global. Por tanto, se trata de una revisión bibliográfica vigente sobre marketing y todo lo referente al estudio de las técnicas de mercadeo.

La revisión se ha centrado en textos, documentos y artículos científicos publicados disponibles en la web, considerando que aquella herencia de la globalización nos permite acceder a mayor y mejor información a través de las Tecnologías de la información. El motor de búsqueda ha sido herramientas académicas de la web que direccionan específicamente a archivos con validez y reconocimiento científico, descartando toda información no confirmada o sin las respectivas referencias bibliográficas.

\section{Resultados}

El rápido crecimiento de la tecnología ha permitido el uso a diario de las herramientas de la comunicación desde el acceso rápido a la información (Internet, televisión por cable y por satélite, entre otras) hasta las creaciones de material visual. (Benko, 2000)

La veloz evolución de la tecnología ha permitido el uso cotidiano de un gran número de herramientas de comunicación, desde las transmisiones rápidas de la información (minitel, Internet, televisión por cable y por satélite, etc.) hasta las creaciones de imágenes. (Benko, 2000)

En esta era todo el mundo se comunica, la gran mayoría de las personas tienen a la mano aunque sea un teléfono inteligente que les permita abrir esa ventana al mundo de la información 


\section{Marketing con enfoque en el crecimiento}

Vol. 2, núm. 1., (2018)

Christian Josuepht Moreno Rodriguez; Ángel Mauricio Chávez Garcés, Mónica Elizabeth Zambrano Moreira

y la tecnología haciendo que los soportes y los canales de información hayan cambiado mucho últimamente, lo que hace que el nacimiento del marketing y los cambios en la comunicación están intrínsecamente ligados. (Benko, 2000)

Todo el mundo se comunica, no es una novedad, pero los métodos, los soportes y los canales de transmisión han cambiado mucho últimamente. No es mi intención entrar aquí en un estudio detallado de la evolución de la comunicación, mas solamente hacer notar que el nacimiento del marketing y las mutaciones en el sector de la comunicación están íntimamente ligados. (Benko, 2000)

Si admitimos que la representación es una creación social y/o individual de un esquema pertinente de lo real, podemos considerar el marketing como una nueva forma de representación geográfica que aporta grandes avances en la economía mundial.

Los elementos básicos del marketing, aquellas de actividades comerciales, de clientes, de competidores, de oferta o de precios, tienen un medio cotidiano para los representantes de las empresas. Parece, esencial aplicar los principales fundamentos de la industria del marketing al campo de la conceptualización: el "marketing territorial", derivado del marketing que al inicio se aplicaba exclusivamente a los productos de gran consumo. (Benko, 2000)

El marketing constituye una nueva etapa en el enfoque de "crecimiento económico" es por ellos que con el uso de técnicas que han tenido como objeto las mejoras en la comercialización y se ha logrado posicionar productos en zonas casi inciertas logrando mayores ventas y superando los objetivos de cualquier empresa o si se quiere dando a conocer lo que queremos abarcando infinitas áreas.

A medida que las empresas implementan disciplina de marketing adoptan un mayor crecimiento económico atrayendo clientes y logrando gran satisfacción entre los consumidores.

Por tanto existe un alto nivel de actividad económica que relaciona al marketing como responsable de esto, haciendo evidente que en nuestra economía nada ocurre hasta que alguien venda algo incrementando una urgente necesidad de un marketing cada vez mayor. 


\section{Marketing con enfoque en el crecimiento}

Vol. 2, núm. 1., (2018)

Christian Josuepht Moreno Rodriguez; Ángel Mauricio Chávez Garcés, Mónica Elizabeth Zambrano Moreira

Como resultado de esto tenemos el aporte de las redes sociales a través de las cuales es másfácil vender una idea o producto debido a la cercanía que tenemos hacia el manejo de las mismas. Estamos al alcance de cualquier información de nuestro interés solo con tomar un teléfono móvil ya tenemos acceso a un sinfín de temas todo gracias al internet y lo que las herramientas tecnológicas nos brindan.

En años anteriores el acceso a la información era más difícil lo que retrasaba el crecimiento de una nación en mayor tiempo.

Hoy en día vender una idea o producto y atraer consumidores es de fácil manejo para nuestra sociedad, la era tecnológica nos aporta estas bondades que nos ayudan el día a día de cada individuo incrementando el crecimiento global, social y económico y brindándonos una mejor calidad de vida a todos.

\section{El nuevo paradigma del marketing}

En la actualidad muchas de las funciones del marketing han sido reorientadas a cargo de la llegada de nuevos conocimientos como Calidad Total, Reingeniería de Procesos, entre otras. Las funciones individuales de los miembros de una organización también están sufriendo transformaciones, al renunciar a tareas o actividades aisladas para crear mayor énfasis en el desempeño de los procesos y su marca en la capacidad de la organización y gratificar las necesidades de sus clientes. Internet como una fuente de acceso libre a la información puede ser utilizada como una parte integrante del concepto moderno de marketing. Al respecto, según Chaffey et al. (2000:5):

- Se convierte en un instrumento de soporte de los distintos procesos y funciones organizacionales e institucionales teniendo que ver con la distribución de productos y servicios a los consumidores.

- Es un medio de comunicación invasivo y poderoso que ayuda a la integración de las diferentes partes funcionales de la organización. 


\section{Marketing con enfoque en el crecimiento}

Vol. 2, núm. 1., (2018)

Christian Josuepht Moreno Rodriguez; Ángel Mauricio Chávez Garcés, Mónica Elizabeth Zambrano Moreira

- Facilita el manejo de la información, la cual es reconocida como una herramienta de marketing para la formulación de estrategias y su implementación.

- El papel futuro de Internet y sus impactos en el mundo empresarial debería formar parte de la visión futura de la organización.

La nueva manera de los medios de comunicación hace que el internet y otros medios digitales faciliten la comunicación interactiva que no es posible a través del marketing convencional. Otros medios que también se incluyen son la televisión digital, los teléfonos móviles, los CD-ROM y los kioskos de información. John Deighton (1996 citado por Chaffey et al. 2000: 17) identifica las siguientes características de la comunicación digital: a) el consumidor inicia el contacto; b) el consumidor busca la información; c) es un medio de alta intensidad, debido a que el consumidor gozará del $100 \%$ de la atención individual cuando esté consultando una web site; d) una empresa puede obtener y almacenar la respuesta de manera individual, a través del uso de bases de datos; y e) las necesidades individuales del consumidor pueden ser atendidas en futuras solicitudes.

De igual manera, Kiani (1998 citado por Chaffey et al. 2000:), ha presentado una serie de diferencias entre el marketing convencional y el nuevo modelo o nuevo paradigma de marketing, según se muestra en Tabla 2.

Tabla 2: Diferencias entre el marketing clásico y el nuevo modelo del marketing

\begin{tabular}{|c|c||c|}
\hline $\begin{array}{c}\text { Marketing } \\
\text { convencional }\end{array}$ & $\begin{array}{c}\text { Nuevo modelo de } \\
\text { marketing }\end{array}$ & Comentarios \\
\hline $\begin{array}{c}\text { Modelo de interacción } \\
\text { uno-a muchos (desde } \\
\text { la compañía a varios } \\
\text { consumidores) }\end{array}$ & $\begin{array}{c}\text { Modelo de interacción } \\
\text { uno-a-uno (desde la } \\
\text { compañía a un sólo } \\
\text { consumidor) o muchos-a- } \\
\text { muchos }\end{array}$ & $\begin{array}{c}\text { Hoffman y Novak (1996) establecen } \\
\text { que, teóricamente, Internet es un } \\
\text { medio de muchos- a-muchos, pero } \\
\text { para las relaciones compañía- } \\
\text { consumidor es mejor considerar el } \\
\text { modelo uno-a-uno }\end{array}$ \\
\hline Marketing masivo & $\begin{array}{c}\text { Marketing } \\
\text { individualizado }\end{array}$ & $\begin{array}{c}\text { a personalización es posible gracias } \\
\text { a que la tecnología permite } \\
\text { monitorear las preferencias y ajustar } \\
\text { el contenido (Deighton, 1996) }\end{array}$ \\
\hline \hline
\end{tabular}




\section{Marketing con enfoque en el crecimiento}

Vol. 2, núm. 1., (2018)

Christian Josuepht Moreno Rodriguez; Ángel Mauricio Chávez Garcés, Mónica Elizabeth Zambrano Moreira

\begin{tabular}{|c|c|c|}
\hline Monólogo & Diálogo & $\begin{array}{c}\text { Indica la naturaleza interactiva de la } \\
\text { World Wide Web con facilidades para } \\
\text { el feedback }\end{array}$ \\
\hline Uso de la marca & Comunicación & $\begin{array}{c}\text { Aumento de la participación del } \\
\text { consumidor en la definición de las } \\
\text { características de la marca. Mayores } \\
\text { oportunidades de agregar valor a la } \\
\text { marca }\end{array}$ \\
\hline $\begin{array}{c}\text { Razonamiento del lado } \\
\text { de la oferta }\end{array}$ & $\begin{array}{c}\text { Razonamiento del lado de } \\
\text { la demanda }\end{array}$ & $\begin{array}{c}\text { La influencia del consumidor es más } \\
\text { importante }\end{array}$ \\
\hline $\begin{array}{c}\text { Consumidor como } \\
\text { objetivo }\end{array}$ & $\begin{array}{c}\text { Consumidor actúa como } \\
\text { socio }\end{array}$ & $\begin{array}{c}\text { El consumidor tiene mayor fuerza en } \\
\text { los productos y servicios requeridos }\end{array}$ \\
\hline $\begin{array}{c}\text { Segmentación } \\
\text { Comunidades virtuales }\end{array}$ & $\begin{array}{c}\text { Agregaciones de consumidores con } \\
\text { inclinaciones similares antes que la } \\
\text { definición de segmentos objetivos }\end{array}$ \\
\hline
\end{tabular}

Fuente: Kiani. 1998 (citado por Chaffey et al. 2000:17) (Anato, 2006).

Los canales de comercialización clásicos y modernos, así como los instrumentos de comunicación más conocidos en turismo, se muestran en la Tabla 3.

\begin{tabular}{|c|c|c|}
\hline \multicolumn{2}{|c|}{ Canales de comercialización } & Instrumentos de \\
\hline Clásicos o Tradicionales & Modernos & \\
\hline $\begin{array}{ll}\text { - } & \text { Agencias de viaje y } \\
& \text { tour operadores } \\
\text { - } & \text { Empresas públicas } \\
& \text { y oficinas de } \\
\text { turismo } & \\
\text { - } & \text { Centrales de } \\
& \text { reserva de los } \\
& \text { prestadores } \\
\text { - Empresa privadas } & \text { Eerá } \\
\text { - } & \text { Venta por catálogo, } \\
& \text { teléfono y } \\
& \text { teletiendas }\end{array}$ & $\begin{array}{ll}\text { - } & \text { Quioscos multimedia } \\
\text { - } & \text { GDS (Amadeus, } \\
& \text { Galileo, } \\
& \text { Sabre, Worldspan, } \\
& \text { etc.) } \\
\text { - } & \text { CDI } \\
\text { - } & \text { Teletexto } \\
\text { - } & \text { Cajeros automáticos } \\
& \text { de billetes } \\
\text { - } & \text { CD-ROM } \\
\text { - } & \text { Videotexto } \\
\text { - } & \text { Internet }\end{array}$ & $\begin{array}{l}\text { - } \text { Identidad corporativa } \\
\text { - } \text { Publicidad } \\
\text { - } \text { Marketing directo } \\
\text { - } \text { Merchandising } \\
\text { - } \text { Forkshops } \\
\text { - } \text { Viajes de familiari- } \\
\text { zación } \\
\text { - Patrocinio o trueque } \\
\text { - Relaciones públicas } \\
\text { - Relaciones con medios } \\
\text { - } \text { de comunicación } \\
\text { Promociones }\end{array}$ \\
\hline
\end{tabular}

Fuente: Valls 2003 y Cerveró 2002 (Anato, 2006). 


\section{Marketing con enfoque en el crecimiento}

Vol. 2, núm. 1., (2018)

Christian Josuepht Moreno Rodriguez; Ángel Mauricio Chávez Garcés, Mónica Elizabeth Zambrano Moreira

El turismo también es abarcado por la influencia del marketing, hoy en día se observan el desarrollo de nuevos tipos de turismo, aumento en las demandas, surgimiento de nuevos destinos, acceso de información de sitios para visitar que antes eran considerados destinos remotos. (Medaglia \& Silveira, 2009)

Se está generando el incremento de otras formas de viajes obligando al turismo y todas las áreas relacionadas a crear transformaciones como consecuencia del progreso de la propia actividad, analizando la evolución del marketing y su atención a los destinos turísticos, enfatizando la relación desequilibrada entre la 'P' de producto y la 'P' de promoción. Al mismo tiempo que apunta su relación con la planificación turística y las ventajas que el marketing de destinos turísticos puede originar. El sendero para lograr este objetivo es un estudio empírico de naturaleza aplicada, ya que, se estudia y analiza la bibliografía relacionada con el tema en un propósito de sugerir la sinergia entre la planificación turística y el marketing de destinos turísticos. (Medaglia \& Silveira, 2009)

\section{Conclusiones.}

En vista de que el marketing abarca tanto el área económica como social tomemos en cuenta que representa un gran impacto a nivel mundial. Gracias a la publicidad y al estudio del mercado podemos llegar a zonas que podrían ser incierta y una infinidad de consumidores.

A medida que las corporaciones y empresas utilicen el marketing como elemento impulsador de ventas y de consumo permiten que las personas tengan un mejor nivel de vida entendiéndoseésto como un crecimiento económico.

Cuando vendemos una idea a través del estado también se está impulsando al crecimiento económico, en el caso de que el estado vende una idea promoviendo la economía solidaria se impulsa a las grandes empresas a tener una responsabilidad social con las pequeñas empresas que están a su alrededor bien sea ofreciendo nuevos productos, aportando a la educación, al acceso a la tecnología, turismo, internet etc, lo que ayuda a la disminución de la pobreza, logrando mayor impacto en el crecimiento de la economía y la calidad de vida. 


\section{Marketing con enfoque en el crecimiento}

Vol. 2, núm. 1., (2018)

Christian Josuepht Moreno Rodriguez; Ángel Mauricio Chávez Garcés, Mónica Elizabeth Zambrano Moreira

Esto incrementa el crecimiento de una nación aportando mejoras y beneficios a sus habitantes a través del ofrecimiento de productos o ideas que satisfacen las necesidades de las personas siendo de su interés y acercándonos a un mayor desarrollo global superando los esfuerzos técnicos, comerciales y de comunicación y permitiendo que el mercado se abra a un desarrollo paulatino en las ventas de ideas y productos, lo que permite el ascenso vertical de las ventas alcanzando elevados porcentajes en un mercado potencial ofreciendo altos beneficios a la sociedad e incrementando el crecimiento de las mismas.

Es debido al impacto socio económico lo que nos lleva a concluir que el marketing es una de las herramientas con mayor potencial que existe en estos tiempos y que logra influenciar el crecimiento en todas las aéreas

En resumen, esta fase se caracteriza por una rentabilidad positiva que debe reinvertirse, en su totalidad, para financiar el crecimiento y los esfuerzos técnicos, comerciales y de comunicación propios de la misma.

\section{Bibliografía.}

Anato, M. (2006). El uso de los instrumentos clásicos del marketing y la tecnología digital en turismo. Estudios y perspectivas en turismo, 16(1), 19-44.

Benko, G. (2000). Estrategias de comunicación y marketing urbano. EURE (Santiago), 26(79), 67-76.

Díaz, A., \& Rubio, R. (2010). Introducción al Marketing. Club Universitario.

Medaglia, J., \& Silveira, C. (2009). La evolución del marketing de destinos: Su sinergia con la planificación turística. Estudios y perspectivas en turismo, 18(5), 530-545. 